\title{
A Microstrip MIMO Antenna with Enhanced Isolation for WiMAX Applications
}

\author{
Nguyen Ngoc Lan, Nguyen Thi Thu Hang, Ho Van Cuu \\ Faculty of Electronics and Telecommunications, Saigon University, Vietnam \\ Correspondence: Nguyen Ngoc Lan, nnlan@moet.edu.vn \\ Communication: received 02 July 2019, revised 09 September 2019, accepted 12 September 2019 \\ Digital Object Identifier: 10.32913/mic-ict-research.v2019.n2.869 \\ The Editor coordinating the review of this article and deciding to accept it was Prof. Nguyen Tan Hung
}

\begin{abstract}
In this paper, a multiple-input multipleoutput (MIMO) antenna with high isolation is designed using defected ground structure (DGS). The proposed antenna is constructed by two sets of four elements $(2 \times 2)$, which are designed at the central frequency of $3.5 \mathrm{GHz}$ for Worldwide Interoperability for Microwave Access (WiMAX) applications. The antenna is fabricated on a FR4 substrate with an overall size of $144 \times 99 \times 1.6 \mathrm{~mm}$. Thanks to DGS, the designed MIMO antenna achieves a high isolation of $30 \mathrm{~dB}$ and a high radiation efficiency of over $90 \%$. Besides, this MIMO antenna attains a $7.5 \mathrm{dBi}$ gain. There is a good agreement between the simulated S-parameters and the measurement results.
\end{abstract}

Keywords: MIMO antenna, mutual coupling, defected ground structure (DGS), microstrip antenna.

\section{INTRODUCTION}

Multiple-output multiple-input (MIMO) is one of the prominent solutions to satisfy the high data rate demand of end users in wireless networks. Employing multipleelement antennas, MIMO can improve both the spectral efficiency and reliability of the transmission without increasing transmitting power or bandwidth [1]. However, when the distance between the antenna elements is not large enough, mutual coupling happens. This is an undesired phenomenon because it not only reduces channel capacity [2], but also introduces extra power loss to the system [3].

Many solutions have been proposed to reduce mutual coupling between antenna elements using, e.g., shorting pins [4], compact coplanar waveguide (CPW) feeding [5], electromagnetic band gap (EBG) [6], parallel coupled-line resonators [7]. These methods have reached a recognizable improvement in isolation enhancement. The isolation between the antenna elements in [4-6] is around $20 \mathrm{~dB}$. The antenna gains are under $2 \mathrm{dBi}$ in [5] and [7]. In addition, the radiation efficiency of the antenna in [5] is $70 \%$. However, these figures can be further improved. In fact, it is challenging to simultaneously optimize multiple parameters, such as the isolation and radiation efficiency, in designing MIMO antennas.

In this paper, we propose a MIMO antenna with enhanced isolation. The antenna is designed at the central frequency $3.5 \mathrm{GHz}$. We apply a defected ground structure (DGS) to achieve a high isolation between the antenna elements of over $35 \mathrm{~dB}$ although the edge-to-edge distance is only $4.3 \mathrm{~mm}$. Based on a FR4 substrate with a thickness of $1.6 \mathrm{~mm}, \varepsilon_{r}=4.4$, and $\tan \delta=0.02$, the antenna has dimension $144 \times 99 \times 1.6 \mathrm{~mm}$. At the central frequency 3.5 $\mathrm{GHz}$, the MIMO antenna reaches over $7.5 \mathrm{dBi}$ gain. The antenna is designed, simulated, and optimized with the Computer Simulation Technology (CST) Studio software. The simulation results are compared with the measurement ones to verify the performance of the proposed antenna.

\section{MiMO Antenna Design}

\section{Design of the Antenna Array}

Our antenna array design is based on the DGS. Figure 1 shows the model of DGS and its equivalent circuit. The DGS is created by connecting two rectangular areas and a microstrip line. The equivalent circuit is made based on the following principle: two rectangles can be considered as an inductance while a microstrip line corresponds to

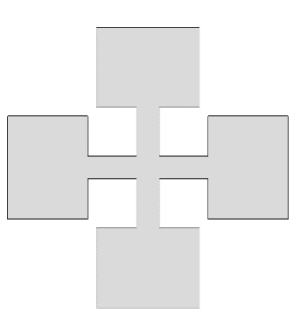

(a) Model

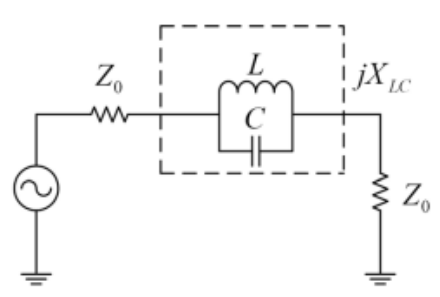

(b) Equivalent circuit
Figure 1. Defected ground structure (DGS) [9]. 


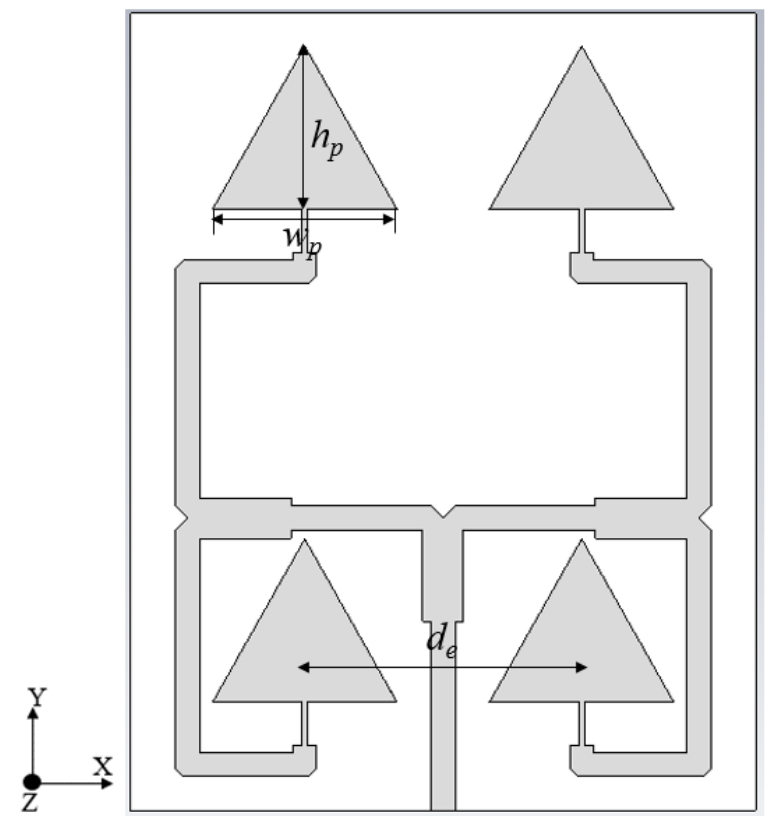

(a) Top view

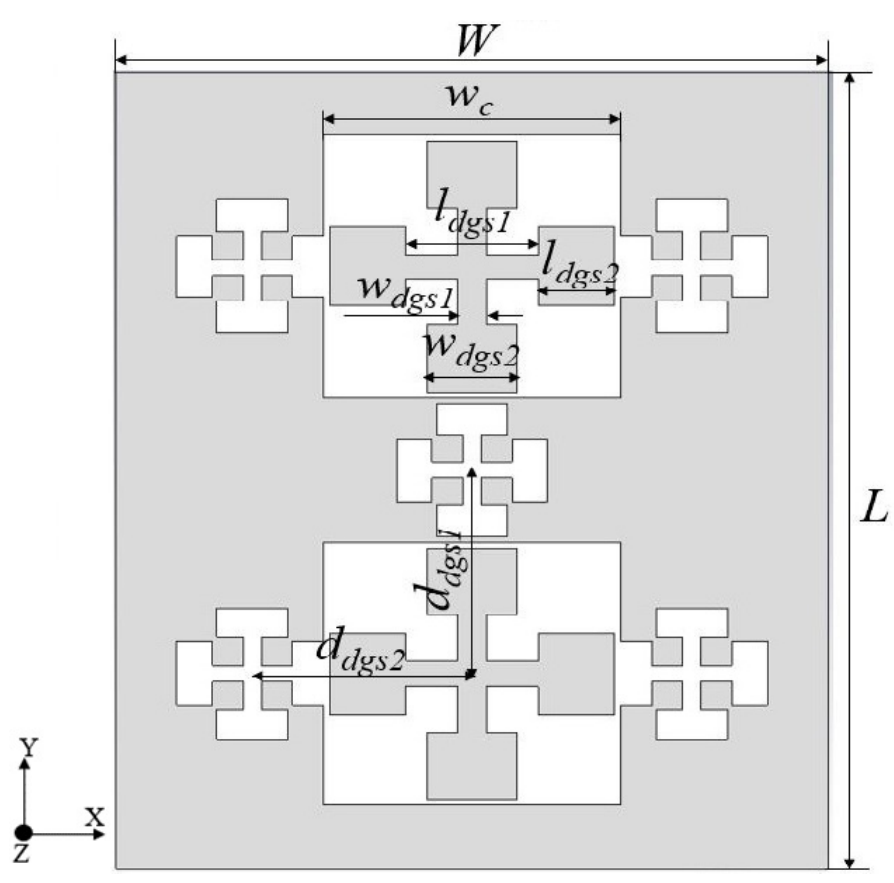

(b) Bottom view

Figure 2. Configuration of proposed DGS-based antenna array.

a capacitance. Therefore, the capacitance and inductance values are given by $[8,9]$

$$
\begin{aligned}
& C=\frac{\omega_{c}}{2 z_{0}\left(\omega_{0}^{2}-\omega_{c}^{2}\right)}, \\
& L=\frac{1}{4 \pi^{2} f_{0}^{2} C},
\end{aligned}
$$

where $Z_{0}$ is the input and output port impedance; $\omega_{0}$ is the angular frequency of the parallel $L C$ resonator, $f_{0}$ is the resonant frequency, and $\omega_{c}$ is the cutoff angular frequency.

Then, the resonant frequency can be calculated as

$$
f_{r}=\frac{1}{2 \pi \sqrt{L C}} .
$$

Next, Figure 2 shows the model of our proposed DGSbased antenna array. The antenna is designed to operate at $3.5 \mathrm{GHz}$ and it is printed on a single layer FR4 substrate with a dielectric constant of 4.4 , a thickness of $1.6 \mathrm{~mm}$, and a loss tangent of 0.02 . The antenna array includes four radiation elements $(2 \times 2)$ and three power dividers on top of the substrate. The ground plane with DGS is placed on the bottom. Using the formula in [10], we can calculate the size of each radiation element. Furthermore, we select equal dividers whose principle can be found in [11]. We use the CST Studio software to optimize and obtain that the dimension of each element is $23.6 \times 20.8 \mathrm{~mm}$ and the distance between elements is approximately $0.4 \lambda$ where $\lambda$ is the wavelength in free space. The overall size of the
TABLE I

DIMENSION PARAMETERS (DEFINED IN FIGURE 2) OF PROPOSED ANTENNA ARRAY

\begin{tabular}{|c|c|c|c|}
\hline Parameter & Value $(\mathrm{mm})$ & Parameter & Value $(\mathrm{mm})$ \\
\hline$W$ & 80 & $L$ & 102 \\
\hline$w_{p}$ & 23.6 & $l_{p}$ & 20.8 \\
\hline$l_{d g s} 1$ & 15 & $w_{d g s 1}$ & 3.2 \\
\hline$l_{d g s} 2$ & 8.5 & $w_{d g s} 2$ & 10.2 \\
\hline$d_{e}$ & 35.4 & $d_{d g s 1}$ & 26 \\
\hline$w_{c}$ & 33.5 & $d_{d g s 2}$ & 24.7 \\
\hline
\end{tabular}

antenna array is $80 \times 102 \mathrm{~mm}$. Table I lists the values of some dimension parameters of the designed antenna array. These parameters are defined in Figure 2.

\section{Design of the MIMO Antenna}

The MIMO antenna consists of two symmetric antenna arrays placed side by side with a separation of $4.3 \mathrm{~mm}$ from edge to edge as illustrated in Figure 3. There are many feeding techniques for antenna such as coaxial feed, coupled, and $\lambda / 4$ impedance transformer [10, 12]. We choose the $\lambda / 4$ impedance transformer in this paper due to its simplicity in impedance matching. Based on a FR4 substrate with a thickness of $1.6 \mathrm{~mm}$, the MIMO antenna has overall size of $144 \times 99 \times 1.6 \mathrm{~mm}$ while the size of one patch is $23 \times 20.125 \mathrm{~mm}$. 


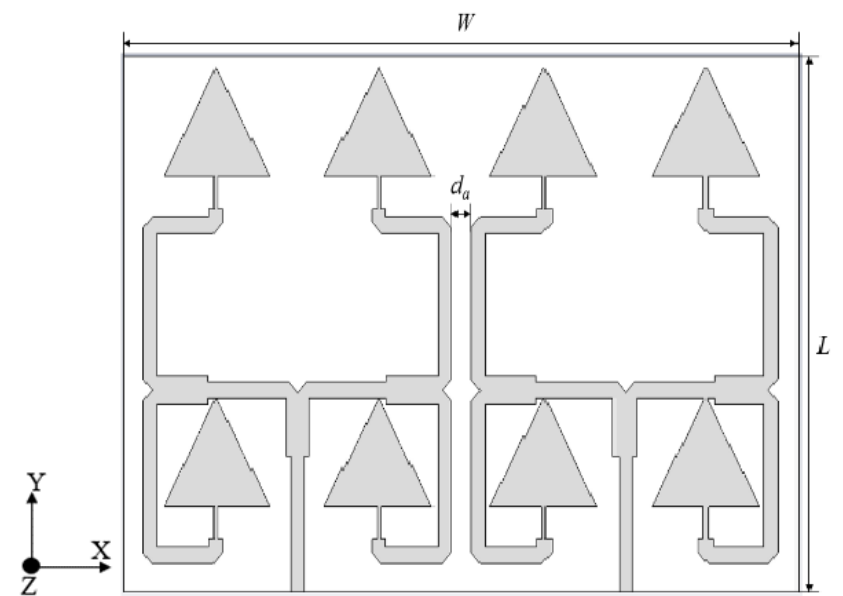

(a) Top view

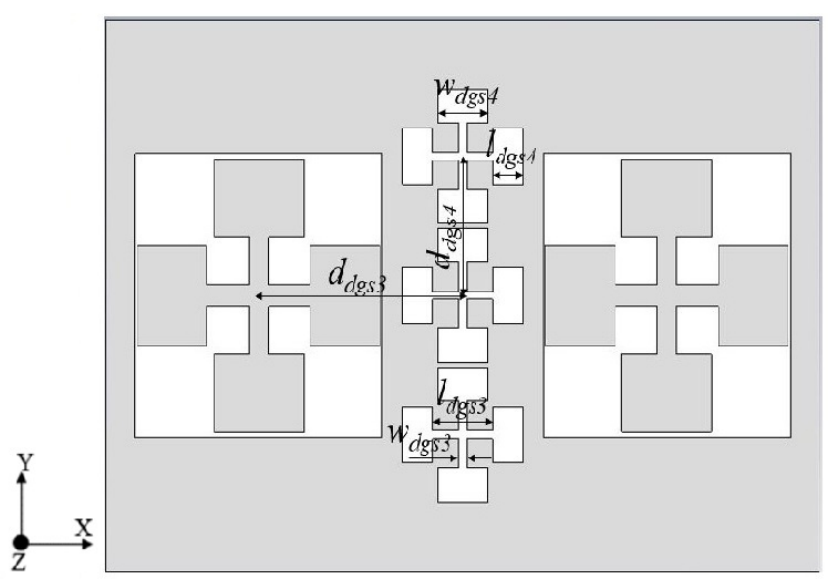

(b) Bottom view

Figure 3. Configuration of proposed MIMO antenna.

TABLE II

DIMENSION PARAMETERS (DEFINED IN FIGURE 3) OF PROPOSED MIMO ANTENNA

\begin{tabular}{|c|c|c|c|}
\hline Parameter & Value $(\mathrm{mm})$ & Parameter & Value $(\mathrm{mm})$ \\
\hline$W$ & 144 & $L$ & 99 \\
\hline$d_{a}$ & 4.3 & $d_{d g s 3}$ & 41.14 \\
\hline$d_{d g s 4}$ & 25 & $w_{d g s 4}$ & 10 \\
\hline$l_{d g s 4}$ & 6 & $w_{d g s 3}$ & 1.5 \\
\hline$l_{d g s 3}$ & 12 & & \\
\hline
\end{tabular}

Table II lists the value of some parameters of the proposed MIMO antenna. These parameters are defined in Figure 3. To reduce the mutual coupling between the elements of the MIMO antenna, we integrate a DGS on the ground plane. The DGS consists of five cells (two large cells and three small cells). To make the capacitance $(C)$ and inductance $(L)$ variable, we use a compensation structure as shown in Figures 1 and 2. This makes a flexibility in optimization.

\section{RESUlts AND Discussions}

\section{Simulation Results}

\section{a) Antenna Array}

Figure 4 presents the reflection coefficients of the proposed antenna array over the frequency band from $2.5 \mathrm{GHz}$ to $4.5 \mathrm{GHz}$. As can be seen, the bandwidth of the antenna is $330 \mathrm{MHz}$, corresponding to $9.4 \%$ of the resonant frequency $3.5 \mathrm{GHz}$. In addition, the antenna has a low return loss of $-30 \mathrm{~dB}$ at the resonant frequency.

Figure 5 shows the $3 \mathrm{D}$ and polar patterns of the proposed antenna array. It can be observed that the the antenna has quite high directivity: it has an angular width $(3 \mathrm{~dB})$ of 40.7 degrees. Moreover, the antenna remains a high radiation efficiency of over $90 \%$.

\section{b) MIMO Array}

As mentioned in Section II.2, the MIMO antenna is integrated with DGS to reduce the effect of mutual coupling. To better understand the effect of DGS on mutual coupling reduction, we compare the S-parameters of the proposed MIMO antenna with and without DGS. The results are displayed in Figure 6. It can be observed that the isolation between antenna elements is significantly improved in the case of DGS. The mutual coupling is $-15 \mathrm{~dB}$ without DGS while with DGS, this figure is less than $-40 \mathrm{~dB}$. This can be explained as follows. Normally, the current distribution in microstrip antenna is uniform without DGS. When there is DGS, the current is redistributed according to the size and position of DGS. By adjusting the size and position of DGS, we can distribute the current at a desired place while limiting the current at other places. This helps us to achieve a high isolation of $40 \mathrm{~dB}$ for the proposed antenna. On top of that, using DGS also improves the bandwidth. We can see that the antenna bandwidth with DGS includes two resonant modes while this value is only one without DGS. As a result, the bandwidth with DGS is larger than without DGS.

Figure 7 shows the pattern of the proposed MIMO antenna. It can be seen that the main lobe direction of the antenna is 190 degrees while the angular width at $3 \mathrm{~dB}$ is 40.7 degrees. Normally, the main lobe direction of a microstrip antenna is 0 degree (uniform current distribution). In our case, utilizing DGS causes a distortion in the current distribution, thus the main lobe direction can be changed. This is a common tradeoff when using DGS. However, given the gain in antenna isolation, this main 


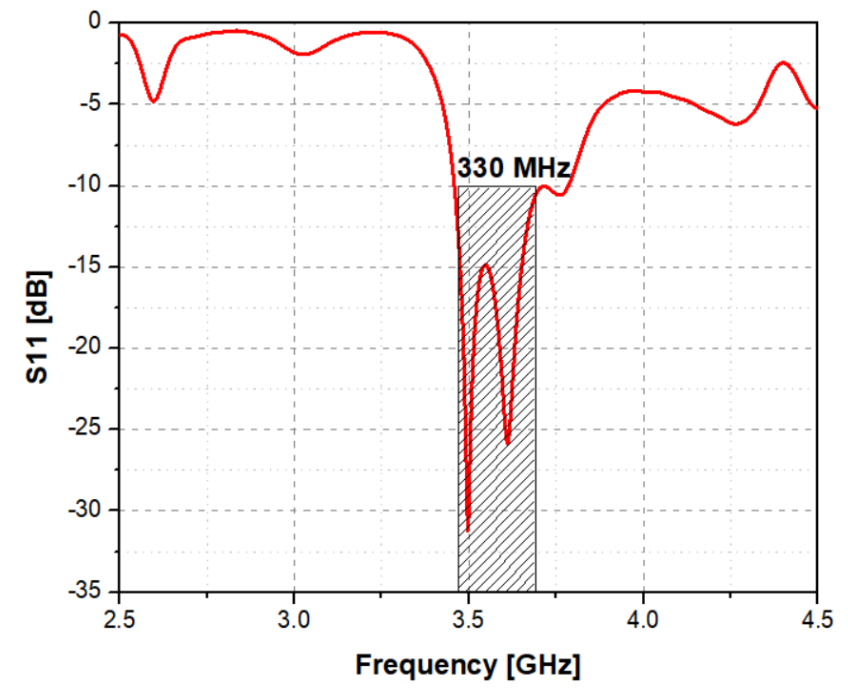

Figure 4. Reflection coefficients of proposed antenna array.

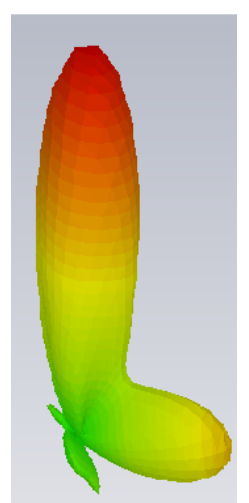

(a) $3 \mathrm{D}$

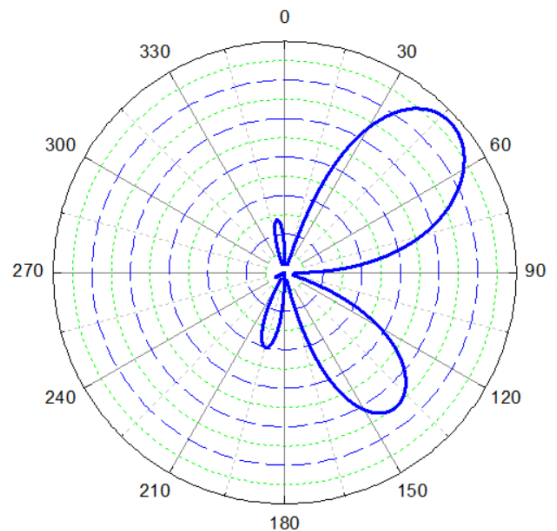

(b) Polar
Figure 5. Pattern of proposed antenna array.

lobe direction change is acceptable. Besides, the gain and radiation of the MIMO antenna reach more than $7.5 \mathrm{dBi}$ and $90 \%$, respectively.

Moreover, in MIMO systems, the independence between radiation patterns of the antennas can be evaluated by the enveloped correlation coefficient (ECC), denoted by $\rho_{e}$. ECC can be calculated from the S-parameters as [13]

$$
\rho_{e}=\frac{S_{11} S_{12}^{*}+S_{21} S_{22}^{*}}{\sqrt{\left(1-\left|S_{11}\right|^{2}-\left|S_{21}\right|^{2}\right)\left(1-\left|S_{22}\right|^{2}-\left|S_{12}\right|^{2}\right) \eta_{1} \eta_{2}}} .
$$

It can also be calculated from the radiation patterns as [14]

$$
\rho_{e}=\frac{\iint_{4 \pi} E_{1}(\theta, \phi) E_{2}^{*}(\theta, \phi) d \Omega}{\iint_{4 \pi} E_{1}(\theta, \phi) E_{1}^{*}(\theta, \phi) d \Omega \iint_{4 \pi} E_{2}(\theta, \phi) E_{2}^{*}(\theta, \phi) d \Omega},
$$

where $E_{1}$ and $E_{2}$ are the far-field radiation patterns, generated from ports 1 and 2 of the antenna while $\theta$ and $\phi$ represents the spherical angles, namely, the elevation and

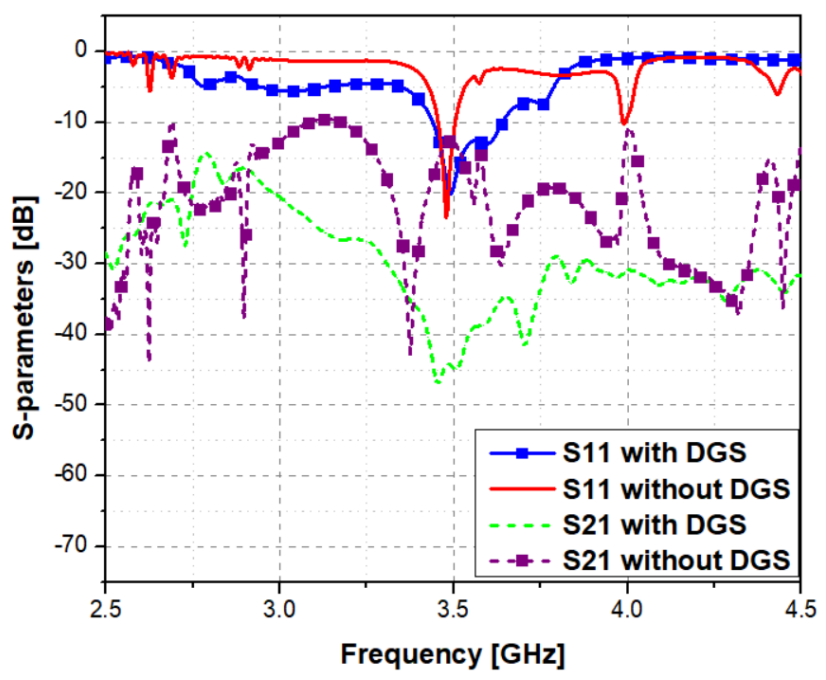

Figure 6. S-parameters of proposed MIMO antenna.

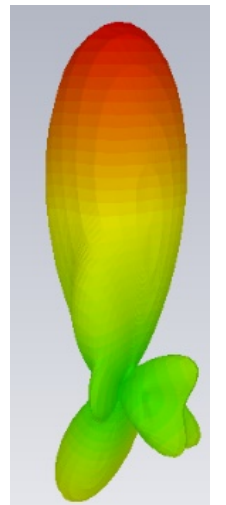

(a) 3D

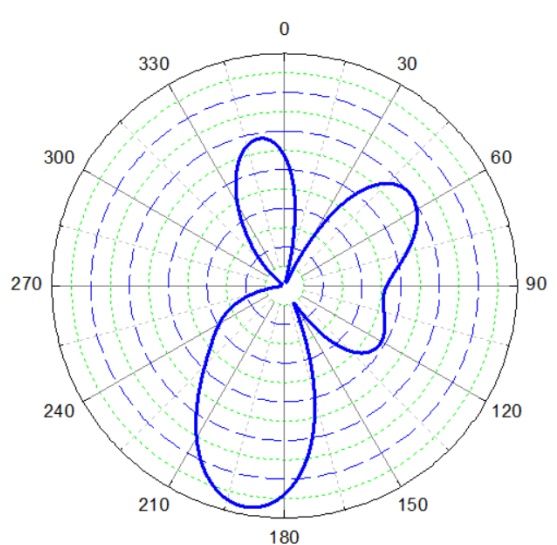

(b) Polar
Figure 7. Pattern of proposed MIMO antenna.

azimuth, respectively. Figure 8 presents the ECC of the proposed MIMO antenna. It is clear that the ECC is smaller than 0.01 from $3.08 \mathrm{GHz}$ to $3.84 \mathrm{GHz}$, corresponding to a band of $760 \mathrm{MHz}$. This is suitable for devices with $\rho_{e} \leq 0.3[15]$.

\section{Measurement Results}

In order to confirm the simulation results by experimental measurements, the prototype of the proposed antenna as shown in Figure 9 is implemented based on a FR4 sheet ( $h=1.6 \mathrm{~mm}, \varepsilon_{r}=4.4$ and $\tan \delta=0.02$ ) with a size of $80 \times 102 \times 1.6 \mathrm{~mm}$ for a single array and $144 \times 99 \times 1.6 \mathrm{~mm}$ for the MIMO antenna.

In Figure 10, we compare the simulated results based on the CST Microwave software and the measurement ones. As can be seen, the measured impedance bandwidths at $-10 \mathrm{~dB}$ of a single array and the MIMO antenna 


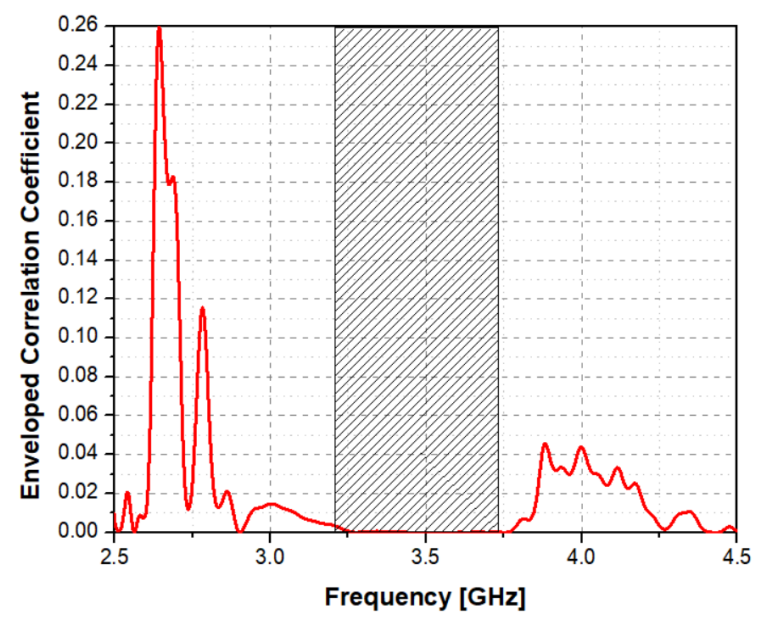

Figure 8. Enveloped correlation coefficient of proposed MIMO antenna.

TABLE III

PERFormanCE COMPARISON OF PROPOSED ANTENNA AND RECENT ANTENNAS

\begin{tabular}{|c|c|c|c|c|}
\hline References & {$[17]$} & {$[18]$} & {$[19]$} & This paper \\
\hline Frequency [GHz] & 2.4 & 3.5 & 2.6 & $\mathbf{3 . 5}$ \\
\hline Bandwidth [\%] & 3.3 & 6.2 & 14.6 & $\mathbf{4 . 1 5}$ \\
\hline Isolation [dB] & 15 & 22 & 15 & $\mathbf{2 9}$ \\
\hline Efficiency [\%] & 85 & 87 & $\mathrm{x}$ & $\mathbf{9 0}$ \\
\hline Gain [dBi] & $\mathrm{x}$ & 3.1 & 4.48 & $\mathbf{7 . 5}$ \\
\hline
\end{tabular}

are $120 \mathrm{MHz}(3.43 \%)$ and $145 \mathrm{MHz}$ (4.15\%), respectively, while the corresponding figures from simulation are $330 \mathrm{MHz}$ and $200 \mathrm{MHz}$, respectively. Moreover, the measured isolation between elements in the MIMO antenna is approximately $30 \mathrm{~dB}$. There is a tolerance between the simulation and measurement results. This can be caused by the instability of parameters in the FR4 substrate [16] and the tolerance in fabrication. However, this tolerance does not affect the operation of antenna and is thus acceptable. To verify the advantage of our proposed antenna, we compare its performance with some previously proposed antenna in the literature in Table III. We can see that the isolation of the antenna in [17] is only $15 \mathrm{~dB}$ and the percentage of the impedance bandwidth is not large $(3.3 \%)$. In [18], although there is a high isolation (22 dB) between array elements, the gain of the antenna is quite low (3.1 dBi). The MIMO antenna in [19] has a large percentage of the impedance bandwidth, but achieves a low gain (4.48 dBi) while the efficiency is not mentioned. In this paper, by using DGS, the proposed antenna achieves a high isolation of approximately $30 \mathrm{~dB}$, and, at the same time, a high efficiency of over $90 \%$. Therefore, despite of the main lobe shift (190 degrees) and a higher complexity when using DGS, our antenna is a promising solution to operate at $3.5 \mathrm{GHz}$.

\section{Conclusion}

This paper presents a MIMO antenna with enhanced isolation for WiMAX applications. The antenna is realized at the central frequency of $3.5 \mathrm{GHz}$ and it is built on a FR4 substrate with parameters: $h=1.6 \mathrm{~mm}, \varepsilon_{r}=4.4$, and $\tan \delta=0.02$. The proposed MIMO antenna contains two sets of $2 \times 2$ elements which incorporates DGS to obtain a high isolation between the elements. From measurements, the antenna achieves approximately $30 \mathrm{~dB}$ in isolation and $90 \%$ in radiation efficiency. Moreover, the antenna has a gain of $7.5 \mathrm{dBi}$ while the measured bandwidth is $145 \mathrm{MHz}$ at $-10 \mathrm{~dB}$. The proposed antenna has a compact size, a planar structure, an easy fabrication, and a low cost, thus can be utilized in practice.

\section{ACKNOWLEDGMENT}

This work is carried out in the framework of the project entitled "Research on methods for mutual coupling reduction between array antenna elements in multiantenna wireless communication system" under Grant number CS2019-38.

\section{REFERENCES}

[1] A. J. Paulraj, D. A. Gore, R. U. Nabar, and H. Bölcskei, 2004, "An overview of MIMO communications - A key to gigabit wireless," in Proceedings of the IEEE, vol. 92, no. 2, pp. 198-217, Feb. 2004.

[2] B. Clerckx, D. Vanhoenacker-Janvier, C. Oestges, and L. Vandendorpe, "Mutual coupling effects on the channel capacity and the space-time processing of MIMO communication systems," in Proc. IEEE International Conference on Communications, Anchorage, AK, USA, June 2003, pp. 2638-2642,.

[3] J. P. Linnartz, "Effects of Antenna Mutual Coupling on the Performance of MIMO Systems," in Proc. 29th Symposium on Information Theory in the Benelux, no. May, 2008.

[4] M. Abdullah, Q. Li, W. Xue, G. Peng, Y. He, and X. Chen, 2019, "Isolation enhancement of MIMO antennas using shorting pins," Journal of Electromagnetic Waves and Applications, vol. 33, no. 10, pp. 1249-1263, 2019.

[5] Duong Thi Thanh Tu, Nguyen Tuan Ngoc, and Vu Van Yem, "Compact Wide-Band and Low Mutual Coupling MIMO Metamaterial Antenna using CPW Feeding for LTE/Wimax Applications," Research and Development on Information and Communication Technology, vol. 2, no. 15, 2018.

[6] N. Kumar and U. Kiran Kommuri, "MIMO Antenna Mutual Coupling Reduction for WLAN Using Spiro Meander Line UC-EBG," Progress In Electromagnetics Research C, vol. 80, Dec. 2018, pp. 65-77.

[7] K. S. Vishvaksenan, K. Mithra, R. Kalaiarasan, and K. S. Raj, "Mutual coupling reduction in microstrip patch antenna arrays using parallel coupled-line resonators," IEEE Antennas and Wireless Propagation Letters, vol. 16, pp. 2146-2149, May 2017.

[8] L. H. Weng, Y. C. Guo, X. W. Shi, and X. Q. Chen, "An Overview on Defected Ground Structure," Progress In Electromagnetics Research B, vol. 7, pp. 173-189, 2008. 

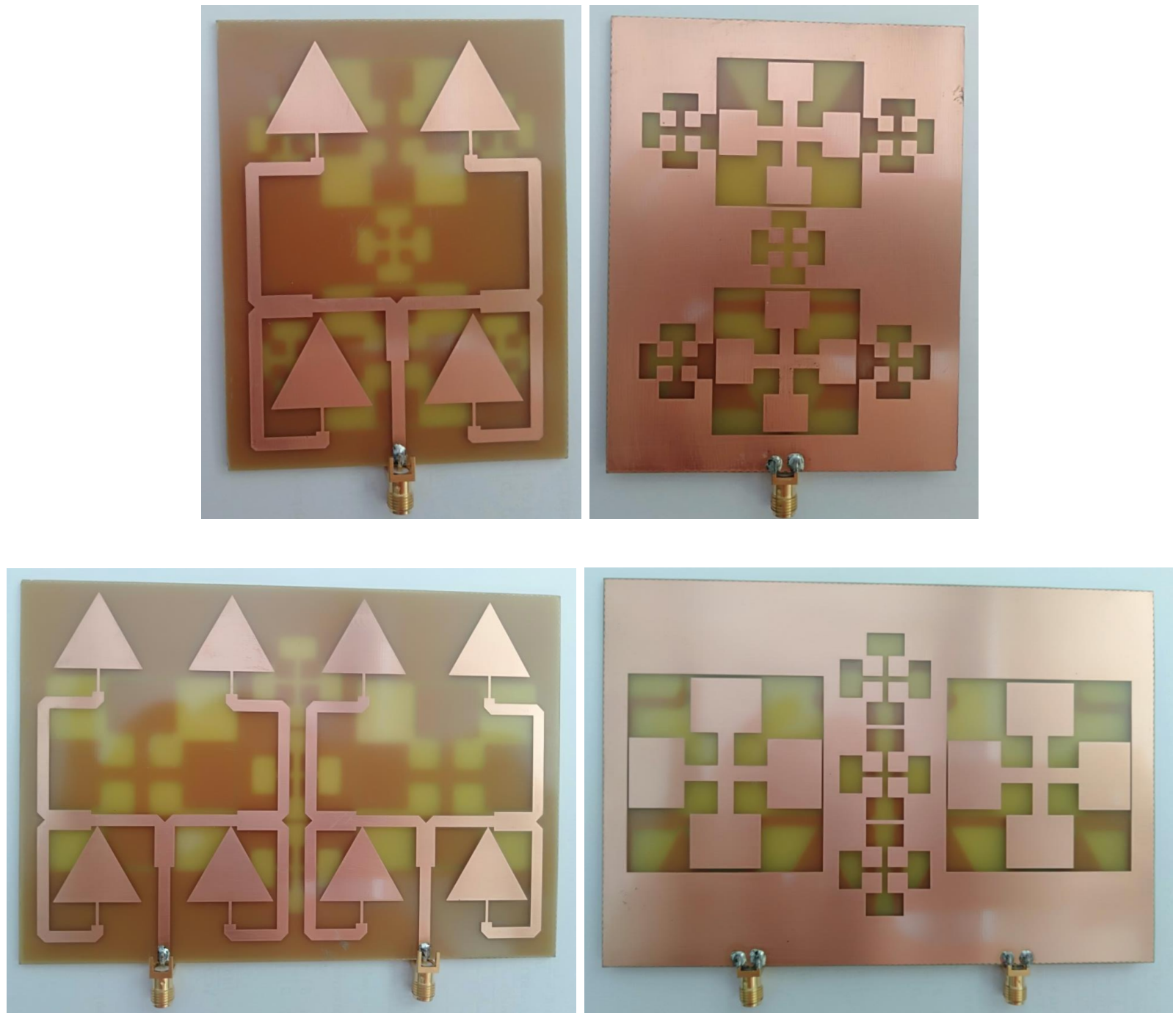

Figure 9. Prototype of fabricated single array (top) and MIMO (bottom) antennas

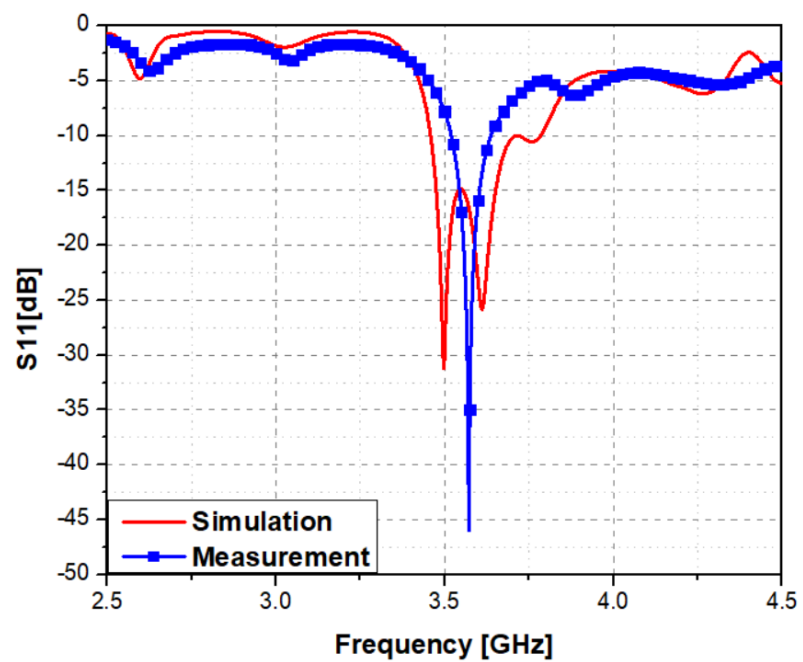

(a) Single array

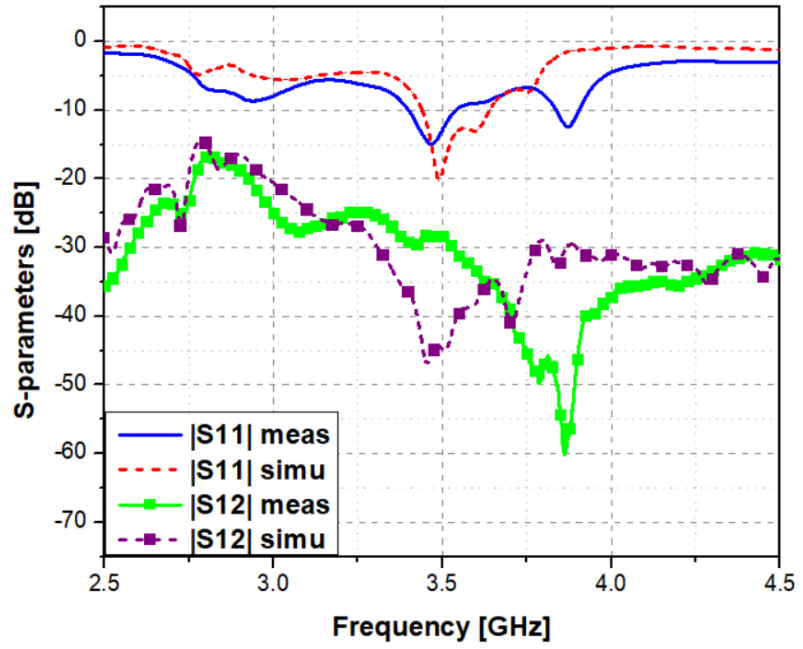

(b) MIMO antenna

Figure 10. The measured and simulated S-parameter results of the proposed antenna. 
[9] T. I. Dal Ahn, Jun-Seok Park, Chul-Soo Kim, Juno Kim, and Yongxi Qian, "A design of the novel lowpass filter using defected ground structure," IEEE Transactions on Microwave Theory and Techniques, vol. 49, no. 1, pp. 86-93, 2001.

[10] Constantine A. Balanis, Antenna Theory: Analysis and Design. United States of America: John Wiley \& Sons, Inc, 2016.

[11] N. L. Nguyen and V. Y. Vu, "Gain enhancement for MIMO antenna using metamaterial structure," International Journal of Microwave and Wireless Technologies, pp. 1-12, no. May, 2019.

[12] A. I. Ramesh Garg, Prakash Bhartia, and Inder Bahl, $M i$ crostrip Antenna Design Handbook. Norwood, MA: Artech House, Inc, 2001.

[13] I. C. S. Blanch and J.Romey, "Exact representation of antenna system diversity performance from input parameter description," Electronics Letters, vol. 39, no. 9, pp. 705-707, 2003.

[14] R. Cornelius, A. Narbudowicz, M. J. Ammann, and D. Heberling, "Calculating the envelope correlation coefficient directly from spherical modes spectrum," in Proc. 11th European Conference on Antennas and Propagation, EUCAP 2017, no. 3, 2017, pp. 3003-3006.

[15] V. 3. 3GPP TS 36.101, EUTRA User Equipment Radio Transmission and Reception. 2008.

[16] L. Peng and C. L. Ruan, "UWB band-notched monopole antenna design using electromagnetic-bandgap structures," IEEE Transactions on Microwave Theory and Techniques, vol. 59, no. 4, PART 2, pp. 1074-1081, 2011.

[17] J. Deng, J. Li, L. Zhao, and L. Guo, "A Dual-Band InvertedF MIMO Antenna with Enhanced Isolation for WLAN Applications," IEEE Antennas and Wireless Propagation Letters, vol. 16, pp. 2270-2273, 2017.

[18] R. Karimian and H. Tadayon, "Multiband MIMO Antenna System with Parasitic Elements for WLAN and WiMAX Application," International Journal of Antennas and Propagation, vol. 2013, pp. 1-7, 2013.

[19] L. Yang, J. Fang, and T. Li, "Compact dual-band MIMO antenna system for mobile handset application," IEICE Transactions on Communications, vol. E98B, no. 12, pp. 2463-2469, 2015.

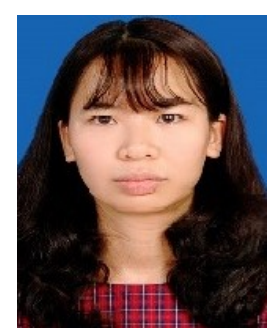

Nguyen Ngoc Lan received the Master and Ph.D. degrees in School of Electronics and Telecommunications, Hanoi University of Science and Technology, Vietnam, in 2014 and 2019, respectively. Currently, she is a lecturer at the Faculty of Electronics and Telecommunications, Saigon University, Vietnam. Her research interests include microstrip antenna, mutual coupling, MIMO antennas, array antennas, reconfigurable antennas, polarization antennas, metamaterial, and metasurface.

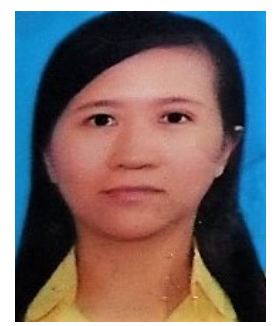

Nguyen Thi Thu Hang received the Bachelor and Master degrees from the Ho Chi Minh City University of Technology, Vietnam, in 1999 and 2002, respectively. Currently, she is a lecturer at the Faculty of Electronics and Telecommunications, Saigon University, Vietnam. Her research interests include digital signal processing, FPGA, and integrated circuit design.

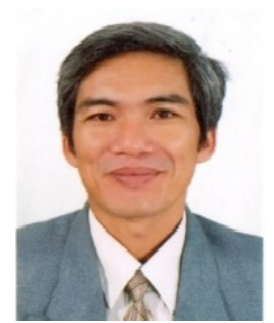

Ho Van Cuu received the Ph.D. degree from the Department Telecommunication, Posts and Telecommunications Institute of Technology, Vietnam, in 2006. Currently, he is a lecturer at the Faculty of Electronics and Telecommunications, Saigon University, Vietnam. His research interests include wireless communications and digital communications. 